\title{
Transmission Electron Microscopy Study of InN Nanorods
}

\author{
Z. Liliental-Weber ${ }^{1}, \mathrm{X} . \mathrm{Li}^{1}$, O.Kryliouk ${ }^{2}$, H.J. Park ${ }^{2}$, J. Mangum ${ }^{2}$, T. Anderson ${ }^{2}$ \\ ${ }^{I}$ Materials Sciences Division, Lawrence Berkeley National Laboratory, Berkeley, CA 94720 USA \\ ${ }^{2}$ Dept. of Chemical Engineering, University of Florida, Gainesville, FL 32611, U.S.A.
}

\begin{abstract}
InN nanorods were grown on a, c-, and r-plane of sapphire and also on $\mathrm{Si}(111)$ and GaN (0001) by noncatalytic, template-free hydride metal-organic vapor phase epitaxy and studied by transmission electron microscopy, electron energy loss (EELS) and photoluminescence (PL) at room temperature. These nanocrystals have different shapes and different faceting depending on the substrate used and their crystallographic orientation. EELS measurements have confirmed the high purity of these crystals. The observed PL peak was in the range of $0.9-0.95 \mathrm{eV}$. The strongest PL intensity was observed for the nanocrystals with the larger diameters.
\end{abstract}

Keywords: Indium nitride nanorods, structure, faceting, TEM studies

PACS: PACS numbers; 61.46.Hk; 68.37.Lp; 79.20.Uv; 78.55.-m

\section{INTRODUCTION}

Major developments in wide-gap III-V nitride semiconductors have recently led to the commercial production of high brightness light emitting diodes (LEDs) with light output from green to near UV. InN has some promising transport and electronic properties. It has the smallest effective electron mass of all the group-III nitrides, which leads to a potentially high mobility and high saturation velocity and a large drift velocity at room temperature. As a result of these properties, there has been a large increase in interest in InN for use in optoelectronic devices, such as laser diodes and high efficiency solar cells, as well as high frequency/high power electronic devices. The growth of high quality epitaxial layers of InN has been established by molecular beam epitaxy and metalorganic chemical vapor deposition. However, the fabrication of potential one-dimensional structures, such as nanowires and nanorods, has proven difficult due to the thermodynamic properties of In and N. Unfortunately, high quality $\mathrm{InN}$ is very difficult to synthesize. It has a very low decomposition temperature and requires a large overpressure of $\mathrm{N}$. There are only a few reports on the one-dimensional synthesis of InN nanostructures.

InN nanorods were successfully grown on c- $\mathrm{Al}_{2} \mathrm{O}_{3}$, $\mathrm{GaN}$, and $\mathrm{Si}$ substrates by non-catalytic, template-free hydride metal-organic vapor phase epitaxy (HMOVPE). Given the challenges in growing $\mathrm{InN}$ structural characterization is required to establish their quality. Transmission electron microscopy (classical and high resolution) together with electron energy loss spectroscopy was applied to learn about structural quality and observe the differences between nanorods grown on different substrates.

\section{EXPERIMENTAL METHODS}

The InN nanorods were grown using a horizontal hot wall H-MOVPE reactor. Trimethyl indium (TMIn) and $\mathrm{NH}_{3}$ were used as In and $\mathrm{N}$ sources, respectively. The growth was performed at atmospheric pressure in $\mathrm{N}_{2}$ ambient. The growth temperature varied from 400 to $700{ }^{\circ} \mathrm{C} ; \mathrm{HCl} / \mathrm{TMIn}$ inlet mole ratio was varied from 0 to $6 ; \mathrm{V} / \mathrm{III}$ ratio ranged from 100 to $10000 ; \mathrm{N}_{2}$ carrier gas flow rate $=1.6 \mathrm{slm}$. The diameter, density and orientation of the nanorods were controlled by growth temperature, substrate selection, and $\mathrm{HCl} / \mathrm{TMI}$ and V/III ratios.

\section{RESULTS}

InN nanorods grown on the c-, a- and r-plane of $\mathrm{Al}_{2} \mathrm{O}_{3}$ are monocrystalline but their shape and faceting differ from each other. The majority of them are elongated along the wurtzite c-axis but not all crystals are distributed vertically to the substrate and thus form flower-like or random arrangement. The nanorods grown on r-plane $\mathrm{Al}_{2} \mathrm{O}_{3}$ are round in cross-section tapering to pencil-shaped at the growth front with a small plateau on the c-plane at the tip. Their diameter and length are in the range of $300-600 \mathrm{~nm}$ and $1000-$ $2500 \mathrm{~nm}$, respectively. The nanorods grown on the cplane and a-plane have a hexagonal cross-section and their diameter is smaller $(100-200 \mathrm{~nm})$ and the length comparable to these grown on a-plane $(700-2000 \mathrm{~nm})$. Usually nanorods grown on c-plane have larger diameters compared to these grown on a-plane. Since 
these nanorods were removed from the substrate for TEM observation they appear rectangular at the one end and with small facets on the other end. It therefore understood that these rectangular shape ends, whose surface is not atomically flat, were earlier attached to the substrate and ends with facets indicate growth direction. Convergent Beam Electron Diffraction (CBED) shows that these facets form about $60^{\circ}$ angle to c-axis arranged along N-polar direction. However, occasionally one can find nanocrystals where faceting is observed for both growth polarities. Facets and most of the tops of the faceted tips are atomically flat. It appears that one side along the length of the crystal is atomically flat and the other has a substantial roughness. In some nanocrystals grown on a-plane their diameter is slightly changing along their length and a $\mathrm{V}$-shape grove appears along one elongated side of the crystal (Fig. 1). CBED patterns show two interconnected identical patterns where c-axis of the one part is rotated by about $60^{\circ}$ toward the other. This coincides with the [0111] direction, which has the same displacement vector as a prismatic stacking fault (PSF). This suggests that PSFs might be present in these nanorods (similarly as in the InN MBE grown layers). PSFs may give rise to the growth of the crystals at different angle and thus the "flower-like" features.

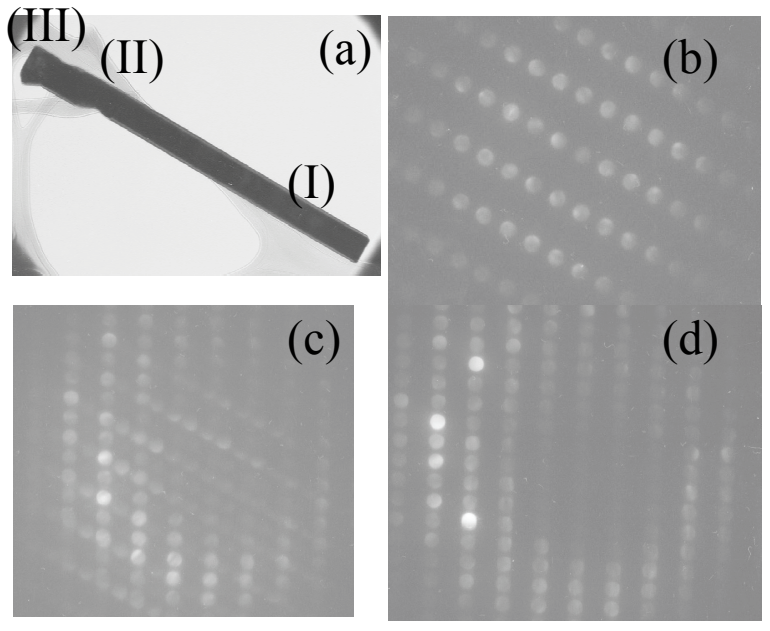

FIGURE 1.(a) A nanocrystal grown earlier on the a-plane of $\mathrm{Al}_{2} \mathrm{O}_{3}$. Note formation of a $\mathrm{v}$-shaped grove along one side of the crystal. (b-d) Convergent Beam Electron Diffraction patterns obtained from the areas indicated by numbers: (b) from area (i); (c) from area (II), and (d) from area (III) showing two interconnected crystals rotated $60^{\circ}$ toward each other.

The shape of nano-crystals grown on $\mathrm{Si}$ and GaN substrate is different. The ones grown on $\mathrm{Si}$ substrate have similar shape to crystals grown on r-plane of $\mathrm{Al}_{2} \mathrm{O}_{3}$. Most of them have a pencil-like shape with slightly asymmetric tips. The side facets form a $30^{\circ}$ angle with the walls of the crystals but their length differs. A small plateau with slightly bend c-planes is present on the top of the tip. Nanocrystals grown on GaN have a shape similar to these crystals grown on cplane sapphire with small facet at their tips and a large, atomically flat plateau.

Since the K-edge of $\mathrm{N}$ is close to the oxygen $\mathrm{K}$ edge Electron Energy Loss Spectroscopy (EELS) was used to verify the nanorods composition and presence of oxygen. There are only few cases where surface oxidation of crystals grown on sapphire can be detected, but majority of them show pure InN composition.

Photoluminescence studies were performed on all crystals. Basically they show the properties of bulk samples, since the nanorod diameters are $\sim 100 \mathrm{~nm}$. The peak position is $\sim 0.9-0.95 \mathrm{eV}$. This corresponds to bulk InN with an electron concentration at mid- $10^{19}$ $\mathrm{cm}^{-3}$.

It is expected that energy position of the PL may depend on the size and quality of the crystal and the electron concentration. The larger the size, the less the surface electron accumulation, and also the lower the electron density, the lower the PL energy and stronger the intensity. Comparing samples on different substrates, the sample grown on Si (111) show Pl at lower energy and much stronger intensity than the three samples grown on sapphire, since it is larger in diameter. Among the samples grown on sapphire, the one grown on r-plane have slightly larger diameter and thus show a stronger PL intensity.

\section{CONCLUSIONS}

TEM studies were performed on InN nanocrystals grown by H-MOVPE. They show high crystalline perfection but some stacking faults are expected to be formed based on high-resolution and CBED studies. The crystals show facet formation on their tips and tips differed in shape depending on type of substrate and crystallographic orientation of the substrate. EELS studies confirm InN purity of the crystals. PL studies show the properties of bulk InN samples since size diameter of these nanocrystals is rather large.

\section{ACKNOWLEDGMENTS}

This work is supported by the Director, Office of Science, Office of Basic Energy Sciences, Division of Materials Sciences and Engineering, of the U.S. Department of Energy under Contract No. DE-AC0205CH11231. The work at UF is partially supported by NSF (CTS-031178) and NASA Kennedy Space Center Grant NAG 10-316, ONR (N00014-98-1-04) and NSF DMR 0400416. 